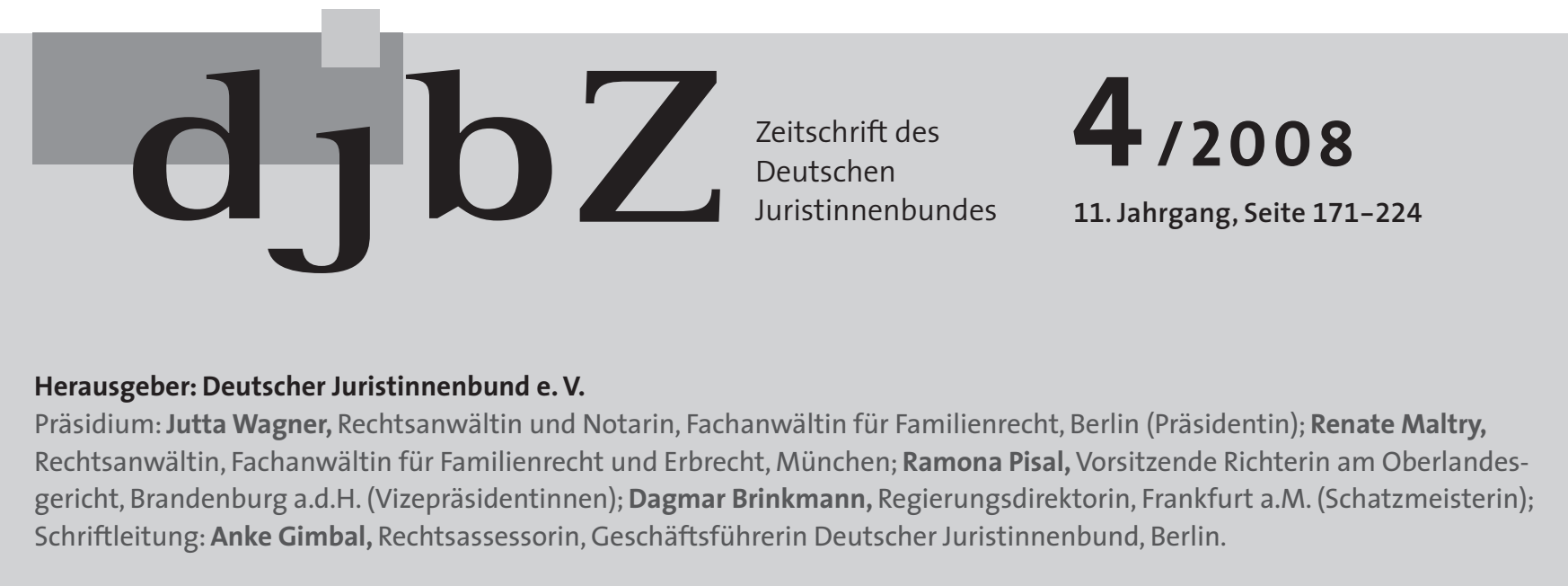

\title{
Die Regelungen zum ehelichen Güterrecht
}

Die Ehe ist zwar grundsätzlich eine nichtvermögensrechtliche Verbindung zwischen zwei Personen, andererseits ergeben sich aus ihr aber soziale und wirtschaftliche Folgen, die es notwendig machen, diesbezügliche Regelungen zu treffen. Mindestinhalt eines ehelichen Güterrechts ist die Bestimmung darüber, wie der familiäre Haushalt finanziert werden soll, wer die Kosten für die Erziehung der Kinder übernimmt, wer die Kosten des Wohnsitzes und wer im Allgemeinen den Unterhalt der Familienmitglieder zu tragen hat. Die Regelungen zum ehelichen Güterrecht sind in Europa höchst unterschiedlich. In den Ländern des Kontinents ist das eheliche Güterrecht in Gesetzen schriftlich niedergelegt, anders in den angelsächsischen Ländern, in denen es reines Richterrecht ist.

Historisch gesehen entstammt die Gütertrennung dem römisch-justinianischen Recht, während zwischen den Germanen ein Güterstand des gemeinschaftlichen Nießbrauchs und der Gesamtverwaltung durch den Ehemann bestand. Die Idee der absoluten Gütergemeinschaft entstammt der christlichen Lehre der engen Gemeinschaft zwischen Mann und Frau. ${ }^{1}$

Das deutsche Recht führte mit dem Bürgerlichen Gesetzbuch von 1896 den Güterstand der männlichen Verwaltung und Nutznießung ${ }^{2}$ ein, eine so genannte Verwaltungsgemeinschaft. Es handelte sich um einen Güterstand der Mitte, der eine schwache Vermögensbindung aufwies. ${ }^{3}$ Dieser Güterstand trat zum 1. April 1953 wegen Verstoßes gegen die Gleichberechtigung außer Kraft. ${ }^{4} 1958$ wurde dann die Zugewinngemeinschaft gesetzlicher Güterstand.
Die gesetzlichen Regelungen der Errungenschaftsgemeinschaft und der Fahrnisgemeinschaft als Wahlgüterstände wurden mit dem GleichberG von 1957 aus dem BGB entfernt, da sie nur selten vereinbart wurden. Dies sollte zur Vereinheitlichung des Güterrechts beitragen. In der DDR wurde mit dem Familiengesetzbuch vom 20. Dezember 1965 die Errungenschaftsgemeinschaft als gesetzlicher Güterstand eingeführt. ${ }^{5}$

Im europäischen Kontext gibt es dem Grunde nach drei große Gruppen des Ehegüterrechts:

1. Güterstände der Gütergemeinschaft,

2. Güterstände der Gütertrennung,

3. Güterstände der Beteiligung an den Zugewinnen.

\section{Güterstände der Gütergemeinschaft}

Güterstände der Gütergemeinschaft kennen Belgien, Dänemark, Spanien, Frankreich, Luxemburg, Italien, Niederlande, Polen, Estland, Tschechien, Slowakei, Portugal und Schweden.

Diese Gütergemeinschaften an sich unterteilen sich aber ihrerseits wieder in drei Untergruppen:

1. Die Gütergemeinschaft an den Errungenschaften,

2. die Gütergemeinschaft des beweglichen Gutes und der Errungenschaften,

3. die universelle Gütergemeinschaft.

1 Nake, Der spanische gesetzliche Güterstand der Errungenschaftsgemeinschaft mit vergleichenden Ausführungen zum deutschen Recht, Diss., S. 65 f. mwN.

2 Beitzke, Familienrecht 1985, S. 94; Staudinger-Thiele, Eheliches Güterrecht, Einl. Rn 6.

3 Soergel-Gaul, Vor 1408, Rn 4.

4 Hierzu Börger, Eheliches Güterrecht, Rn 51.

5 Siehe hierzu auch Artikel von Christiane Lang im gleichen Heft, S. 176.

\section{Dr. Angelika Nake}

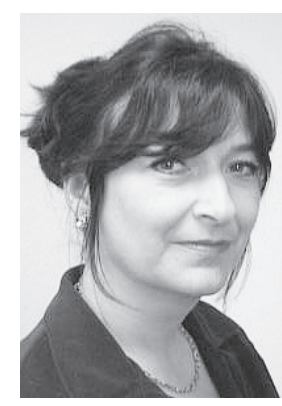

Vorsitzende der Kommission Zivil-, Familien- und Erbrecht, Recht anderer Lebensgemeinschaften des djb, Rechtsanwältin und Fachanwältin für Familienrecht, Darmstadt 


\section{Die Errungenschaftsgemeinschaft}

Sie gehört grundsätzlich zu den Güterständen der Gütergemeinschaft. Man unterscheidet hier üblicherweise zwei Gütermassen, die privaten Güter und das Gemeinschaftsgut bzw. Gesamtgut.

In der Errungenschaftsgemeinschaft bestehen drei Vermögensmassen nebeneinander:

- das jeweilige private Vermögen der Ehegatten,

v die Errungenschaft als eigenständige Masse.

Die Errungenschaftsgemeinschaft ist gesetzlicher Güterstand in Spanien, Italien, Belgien, Frankreich, Luxemburg, Tschechien, Estland und Portugal. Im Folgenden wird in der Regel Bezug genommen auf die Errungenschaftsgemeinschaft des spanischen Rechts oder des französischen Rechts, da ein allgemeiner Vergleich sehr schwierig ist.

Nach der spanischen Codigo Civil sind gemeinschaftliche Vermögensgegenstände gemäß Art. 1347 CC:

1. Die durch die Arbeit oder das Gewerbe eines der Ehegatten erzielten.

2. Die Früchte, Erträge und Zinsen sowohl der privaten Güter, wie auch des gemeinschaftlichen Vermögens.

3. Die entgeltlich auf Kosten des Gesamtgutes erworbenen Güter, sowohl bei Erwerb für die Gemeinschaft wie für nur einen der Ehegatten.

4. Die aufgrund eines Rückkaufsrechts mit Errungenschaftscharakter erworbenen, auch wenn dies mit Mitteln des privaten Vermögens geschieht. Die Gemeinschaft schuldet dann dem Ehegatten den bezahlten Betrag.

5. Die während des Bestehens der Errungenschaftsgemeinschaft durch einen der Ehegatten auf Kosten des Gesamtgutes gegründeten Unternehmen und Betriebe. Stammt das eingesetzte Kapital sowohl aus dem gemeinschaftlichen wie aus dem privaten Vermögen, so sind die Gemeinschaft und die jeweiligen Ehegatten Miteigentümer (Art. 1354 CC).

6. Weiterhin gibt es die Vermutung, dass es sich um Güter des Gesamtgutes handelt, es sei denn, der Beweis des Gegenteils gelingt.

Daneben bestehen jeweils die privaten Güter des Ehegatten, zu denen lt. Art. 1346 CC gehört:

1. Vermögensgegenstände und Rechte, die ihm bei Beginn der Gemeinschaft gehörten,

2. Vermögensgegenstände und Rechte, die er später unentgeltlich erwirbt,

3. Vermögensgegenstände und Rechte, die er auf Kosten oder als Ersatz von privatem Vermögen erwirbt,

4. Vermögensgegenstände und Rechte, die aufgrund eines nur einem der Ehegatten zustehenden Rückkaufrechts erworben wurden,

5. persönliche, unter Lebenden nicht übertragbare Vermögensrechte,

6. Ersatz für Personenschäden eines Ehegatten oder Schäden an seinem privaten Vermögen,

7. Kleidungsstücke und Gegenstände des persönlichen Gebrauchs ohne außergewöhnlichen Wert,
8. die für die Ausübung eines Berufs oder Gewerbes erforderlichen Gegenstände, soweit diese nicht Bestandteil oder Zubehör eines gemeinschaftlichen Unternehmens oder Betriebes sind.

\section{Die reinen Gütergemeinschaften}

Neben der Errungenschaftsgemeinschaft gibt es noch die universelle Gütergemeinschaft, das heißt mit der Heirat werden sämtliche Güter der beiden Ehegatten Gesamtgut. Damit fallen auch Erbschaften und Schenkungen und das gesamte voreheliche Vermögen in das Gesamtgut. Die universelle Gütergemeinschaft ist in vielen Ländern so genannter Wahlgüterstand. Auch in Deutschland. Gesetzlicher Güterstand ist sie nur in den Niederlanden.

\section{Die Güterstände der Gütertrennung}

\section{Die Gütertrennung}

Diese Güterstände finden sich vom Grundsatz her in Großbritannien, Österreich, Schottland, Finnland, Griechenland, Irland und Lettland. Tragend ist hier das Prinzip der Unabhängigkeit der Ehegatten und der Individualität. Der Güterstand der Gütertrennung ist zugeschnitten auf die Ehen, in welchen zwei junge Ehegatten berufstätig sind und beide ihren Lebensunterhalt eigenständig verdienen können.

\section{Die Zugewinngemeinschaft}

Sie ist gesetzlicher Güterstand nur in Deutschland. ${ }^{6}$ Es gibt aber einige Länder, welche die Zugewinngemeinschaft als Wahlgüterstand kennen, so z.B. Spanien. Als gesetzlicher Güterstand stellt sie aber europaweit einen Alleingang dar. Sie basiert im Wesentlichen auf dem Grundsatz der Gütertrennung. Abgesehen von einigen Schutzvorschriften z.B. dem Verkauf des ganzen Vermögens eines Ehegatten, kann jeder Ehegatte sein Vermögen in der Ehe verwalten und verbrauchen wie er will, solang er den Unterhalt der Familie sicherstellen kann. Erst mit der Auflösung der Ehe realisiert sich der Zugewinn, nämlich dass, was ein Ehegatte in der Ehe hinzuerworben hat und es entsteht ein Anspruch auf die Hälfte dieses Zugewinns für den Ehegatten, der weniger erworben hat.

\section{Die Verwaltung des Gesamtguts in den einzelnen Güterständen}

\section{Die Verwaltung des Gesamtgutes}

In den reinen Güterständen der Gütergemeinschaft liegt die Verwaltung des Gesamtgutes grundsätzlich in den Händen der beiden Ehegatten.

Bei den Güterständen der Errungenschaftsgemeinschaften kann es sich unterscheiden zwischen den privaten Gütern und dem Gesamtgut. Häufig ist es so, dass die beiden Ehegatten

6 Die Schweiz versucht mit dem Güterstand der Errungenschaftsbeteiligung eine Art. Mischgüterstand, siehe ZGB 197, 198. 
das Gesamtgut gemeinsam verwalten. Mit einem Ehevertrag ist diese Regelung zu ändern. Alle Verwaltungsmaßnahmen, die die Errungenschaftsgüter betreffen, müssen also gemeinschaftlich getroffen werden. Zu diesen Gütern gehören auch die, die nicht den privaten Gütern zugeordnet werden können oder für die die private Herkunft des Kapitals, mit dem sie gekauft worden sind, nicht nachgewiesen werden konnte. Im Zweifel gilt also für diese Güter die gemeinschaftliche Verwaltung durch beide Ehegatten.

Sinn dieser Regelung ist es, das gemeinschaftliche Handeln der Ehegatten zu fördern und dem nicht handelnden jedenfalls ein Kontrollrecht der Verwaltung des anderen zu geben. In Spanien leitet man dies beispielsweise aus dem Gleichheitsgrundsatz der Verfassung ab, Art. 32 Abs. 1 der spanischen Verfassung. Der Gleichheitsgrundsatz in der Verfassung macht es nach Ansicht des spanischen Rechts notwendig, dass die Ehefrau hierdurch die größtmöglichen Mitwirkungs- und Kontrollrechte hat. Die Berechtigung zur Mitverwaltung in den Güterständen der Gütergemeinschaft erlaubt jedem Ehegatten, im Rahmen der Normen seines jeweiligen Güterstandes, als Anspruchsinhaber oder Anspruchsmitinhaber an der Verwaltung und Verteidigung der gemeinschaftlichen Güter und Rechte teilzunehmen. Die Verwaltungs- und Verfügungshandlungen eines jeden Ehegatten binden die Gemeinschaft. Eine Vertretung oder Bevollmächtigung, auch pauschal für eine Mehrzahl an Geschäften ist möglich, nach dem spanischen Recht bedürfen entgeltliche Verfügungen über die Errungenschaftsgüter grundsätzlich des Einverständnisses beider Ehegatten, gleichgültig welcher Art. die Güter sind. Hierdurch soll gewährleistet werden, dass die wesentlichen Entscheidungen des ehelichen $\mathrm{Zu}$ sammenlebens von beiden Ehegatten gemeinsam getroffen werden. Hierzu gehören neben den Entscheidungen über die Errungenschaftsgüter auch die Wohnsitzwahl, die Erziehung der Kinder und die Art. und Weise des Familienzusammenlebens.

Unentgeltliche Verfügungen sind ohne das Einverständnis des anderen Ehegatten grundsätzlich nichtig. Hiervon ausgenommen sind nur übliche Schenkungen, die aus einer sozialen oder moralischen Pflicht herrühren, z.B. Geburtstagsgeschenke für Angehörige oder enge Freunde.

Die Berechtigung der Mitverwaltung erlaubt jedem Ehegatten, als Anspruchsinhaber oder Anspruchsmitinhaber an der Verwaltung und Verteidigung der ehelichen Güter teilzunehmen. Die Mitverwaltung bedeutet nicht notwendigerweise die Gesamtverwaltung der Güter, sie kann auch die Kontrolle der Verwaltung sein. Als Minimum bedeutet sie aber wenigstens die Miteigentümerschaft an den Gütern.

\section{Die Verwaltung der privaten Güter}

Grundsätzlich können beide Eigentümer im Rahmen der Errungenschaftsgemeinschaften über ihre privaten Güter frei verfügen. Ausnahmen gelten beispielsweise in Spanien und Frankreich für die Familienwohnung und den Hausrat. In der grundsätzlichen Freiheit der Verwaltung der Güter findet sich kein Unterschied zum deutschen Güterstand der Zugewinngemeinschaft, allerdings gibt es im deutschen Recht keinen
Schutz für die Familienwohnung. Diese kann vom Eigentümer nach deutschem Recht verkauft werden, es sei denn, sie stellt das ganze Vermögen des Ehegatten dar.

\section{Die Verwaltung der Güter in der Zugewinngemeinschaft}

Hinsichtlich der Vermögensverwaltung der Zugewinngemeinschaft gilt der $\$ 1364$ BGB. Er bestimmt, dass jeder Ehegatte sein Vermögen alleine verwaltet. Beschränkungen der Verwaltung ergeben sich nur aus den $\mathbb{S} \mathbb{S} 1365$ ff. BGB. Ein Ehegatte kann ohne Zustimmung des anderen nicht über sein Vermögen als Ganzes bestimmen, noch über Haushaltsgegenstände. Ansonsten ist er in seiner Verwaltung aber völlig frei. Es gibt in der Zugewinngemeinschaft auch keinerlei Kontrollrechte über die Verwaltung des Vermögens durch den anderen Ehegatten. Der Auskunftsanspruch, im Rahmen dessen ein Ehegatte über den Bestand seines Vermögens Auskunft erteilen muss, entsteht erst bei der Scheidung der Ehegatten. Während der Ehe gibt es lediglich ein Unterrichtungsanspruch über das Vermögen in groben Zügen. Auch der Auskunftsanspruch nach der Ehe hat insoweit Mängel, als es im Gegensatz zum Unterhaltsrecht keinen Anspruch auf Vorlage von Belegen gibt. Geschuldet wird gemäß $\mathbb{1 3 7 9}$ BGB nur die Vorlage eines Verzeichnisses und nicht die Vorlage von Belegen oder Unterlagen. ${ }^{7}$ Der Ausgleichsberechtigte, also der, der den geringeren Zugewinn hat - in der Regel der Ehegatte, der wegen Kindererziehung zum Teil oder ganz aus dem Berufsleben ausgeschieden ist - hat hier ein ganz erhebliches Beweisproblem.

Eine Einflussmöglichkeit während der Ehe auf die Verwaltung des Vermögens von einem Ehegatten fehlt in der Zugewinngemeinschaft völlig. Erst wenn ein Ehegatte wegen Vermögensverschwendung unter Betreuung gestellt werden muss, hat der andere Ehegatte die Möglichkeit, die Verwaltung als möglicher Betreuer zu übernehmen.

\section{Vergleichende Ausführungen}

Das zentrale Problem der Errungenschaftsgemeinschaft liegt in der gemeinschaftlichen Verwaltung der Güter. Mitverwaltung heißt, dass beide Ehegatten die Handlung gemeinsam vornehmen müssen oder einer unter der Kontrolle des anderen. Der andere Ehegatte handelt dann nicht selbst, er trägt die Handlung aber mit. Auch im spanischen Recht ist beispielsweise die Zustimmung des anderen Ehegatten bei der Verwaltung der eigenen Güter in bestimmten Fällen vorgesehen. So z.B., wenn ein Ehegatte über die in seinem Eigentum stehende Familienwohnung oder den Hausrat verfügt. Er bedarf dann des Einverständnisses des anderen Ehegatten.

Das französische Recht hat sich demgegenüber bei der Errungenschaftsgemeinschaft für eine getrennte Verwaltung der Güter entschieden, ganz so wie im deutschen Recht auch. Es

7 Dies wird sich aber jetzt endlich mit der Reform des deutschen Güterrechts ändern, siehe hierzu auch die Stellungnahme des djb, http://www.djb.de/Kommissionen/kommission-zivil-familien-underbrecht/St_08-02_Zugewinnausgleich und die Pressemitteilung hierzu, http://www.djb.de/Kommissionen/kommission-zivil-familien-und-erbrecht/PMo8-11. 
gilt hier der Grundsatz der sogenannten „konkurrierenden Verwaltung “ der Güter, beide Ehegatten verwalten das Gesamtgut allein; nur in schwerwiegenden Geschäften, die wichtige Teile des Ehevermögens betreffen, greift die gemeinschaftliche Verwaltung ein. Dies hat insofern Vorteile, als hier im Grundsatz jeweils eine Einzelverwaltung vorliegt, durch die eine größere Beweglichkeit und Praktikabilität zugunsten des Rechtsverkehrs erreicht wird. Andererseits vergrößert sich das Risiko, dass beide Ehegatten widerstreitende Verfügungen über dieselbe gemeinschaftliche Sache treffen. Auch das italienische Recht hat sich für die grundsätzliche Alleinverwaltung der Gesamtgüter entschieden, auch hier wird nur für schwerwiegende Rechtsgeschäfte die Mitverwaltung des anderen Ehegatten verlangt.

Wenn bei einem Akt der Verwaltung die Zustimmung des anderen Ehegatten vorgeschrieben ist und dieser sie nicht erteilt, kann das Gericht die Zustimmung ersetzen. Bei unentgeltlichen Verfügungen ist diese Ersetzung allerdings in der Regel nicht möglich.

Auch im spanischen Recht gibt es die sogenannte Schlüsselgewalt. Gemäß Art. 1319 CC kann jeder der Ehegatten die laufenden Geschäfte zur Deckung der gewöhnlichen Bedürfnisse der Familie wahrnehmen, die seiner Obhut anvertraut sind, abhängig von den familiären Umständen und den örtlichen Gebräuchen. Hier deckt sich der Begriff mit dem deutschen Recht der Schlüsselgewalt, $\mathbb{} 1357$ BGB berechtigt jeden der Ehegatten, die Geschäfte zur angemessenen Deckung des Lebensbedarfs der Familie mit Wirkung für und gegen den anderen Ehegatten abzuschließen. Die Geschäfte, die der Ehegatte vornimmt, müssen ihm übertragen sein.

Unterschiedlich geregelt in den beiden Rechtsordnungen ist allerdings die Haftung für diese Handlungen. Während nach deutschem Recht beide voll haften, haftet im spanischen Recht der nicht handelnde Ehegatte nur subsidiär. Die spanische Regelung ist meines Erachtens für den nicht handelnden Ehegatten gerechter und für den Dritten und Gläubiger nicht mit weniger Schutz verbunden.

Im Rahmen des deutschen Rechts ergibt sich weiterhin das Problem, dass die „Nurhausfrau“ kein eigenes Einkommen hat und insoweit keine Lasten tragen kann. Im spanischen Recht ist die Ehefrau, auch wenn sie „Nurhausfrau“ ist, Miteigentümerin der Errungenschaft und hat somit eigene Güter.

Wenn der deutsche Ehegatte kein eigenes Einkommen hat, hat er als Haushaltsführender einen Anspruch auf ein angemessenes Wirtschaftsgeld und einen Anspruch auf ein eigenes Taschengeld, welches etwa bei 5-7 Prozent des Nettoeinkommens liegt. Bei grundsätzlicher Verweigerung dieser Beträge besteht ein Klagerecht für den haushaltsführenden Ehegatten. Will der haushaltsführende Ehegatte aber nicht klagen, so ist er in einer äußerst schlechten Situation. Die spanische Familienfrau oder der spanische Familienmann ist demgegenüber in einer erheblich besseren Situation, da sie/er durch das Miteigentum eigene Güter besitzt.

Der Vorteil der konkurrierenden Verwaltung, für die sich die französische und die italienische Rechtsordnung entschie- den haben, liegt in der größeren Beweglichkeit und Praktikabilität der Verwaltungsmaßnahmen. Als Vorteil der spanischen Lösung wird im spanischen Recht auf den Grundsatz der Gleichberechtigung zwischen Frau und Mann hingewiesen. Es würde gegen den Gleichberechtigungsgrundsatz verstoßen, wenn der Ehemann die gemeinsamen Güter alleine verwalten dürfte, ohne dass die Ehefrau hier ein Mitspracherecht hätte. ${ }^{8}$ Dies kann allerdings auch im Rahmen der konkurrierenden Verwaltung sichergestellt werden. Das französische Recht hat im Jahre 1985 von der Verwaltung des Ehemannes auf die konkurrierende Verwaltung umgestellt.

Das belgische Ehegüterrecht hat bereits im Jahre 1976 die konkurrierende Verwaltung eingeführt, ohne dass es zu einer Zunahme der einschlägigen Streitsachen geführt hätte.

\section{Vergleich zwischen den beiden Güterständen der Errungen- schaftsgemeinschaft und der Zugewinngemeinschaft}

Die deutsche Zugewinngemeinschaft kennt kein Gesamtgut, alle ehelichen Güter verbleiben gem. $\mathbb{} 1363$ BGB im Eigentum desjenigen Ehegatten, der sie mit in die Ehe gebracht hat oder der sie erwirtschaftet hat. Erst mit der Auflösung der Ehe wird errechnet, ob und in welcher Höhe jeder der Ehegatten einen Zugewinn erwirtschaftet hat, d.h. ob er zum Ende der Ehe mehr Vermögen hat, als er zu Beginn der Ehe hatte. Dieser Zugewinn wird dann zwischen den Ehegatten aufgeteilt. Die Zugewinngemeinschaft ist insoweit nicht auf die laufende Ehe zugeschnitten, sondern realisiert sich bei deren Auflösung.

Jeder der Ehegatten kann über seine Güter frei verfügen, \1364 BGB.

Es bestehen lediglich zwei Verfügungsbeschränkungen. Gemäß $\$ 1365$ BGB kann der Ehegatte nicht über sein Vermögen als Ganzes verfügen und gemäß $\ 1369$ BGB besteht eine Verfügungsbeschränkung hinsichtlich der Haushaltsgegenstände. Eine Vorschrift ähnlich dem Art. 1320 CC, dass ein Ehegatte nicht über die in seinem Besitz befindliche Familienwohnung verfügen kann, gibt es im deutschen Recht nicht. Der deutsche Alleineigentümer kann also über die Familienwohnung frei verfügen, selbst wenn die Familie hierdurch obdachlos wird. ${ }^{9}$

Die Vorteile des deutschen Güterstandes zeigen sich bei der Ehe von zwei berufstätigen Ehegatten ohne Kinder (DINKs). Im Rahmen der Zugewinngemeinschaft hat jeder der Ehegatten völlige Freiheit in der Verwaltung seines Vermögens. Auch die Schuldenhaftung ist wesentlich einfacher als in der Errungenschaftsgemeinschaft. Insbesondere im Rahmen der Vermutung des $\mathbb{} 1362$ BGB kann der Gläubiger eines Ehegatten in jedes Gut vollstrecken, das sich im Besitz beider befindet. Er muss auch nicht beide Ehegatten verklagen, die Klage nur gegen den Schuldner-Ehegatten reicht völlig aus.

8 Rams Albesa, La sociedad de gananciales, S. 273.

9 Mit der Einführung eines deutsch-französischen Wahlgüterstandes könnte sich dies demnächst ändern. Hier wird auch für deutsche Ehepaare die Möglichkeit eröffnet, eine Zugewinngemeinschaft zu wählen, die den Schutz der Familienwohnung vorsieht, allerdings bisher die Regelung des $\$ 1369$ BGB nicht aufgenommen hat. 
Die Vorteile der unabhängigen Vermögensverwaltung zeigen sich auch bei einer unternehmerischen Tätigkeit des Ehegatten.

Die Nachteile der Zugewinngemeinschaft zeigen sich aber bei einer Ehe, in welcher nur einer berufstätig ist und der andere die Kinder versorgt. So ist der alleinverdienende Ehegatte nach Abgeltung seiner Unterhaltspflichten völlig frei in seinen Vermögensdispositionen. Der haushaltsführende Ehegatte hat zwar einen Anspruch auf ein angemessenes Wirtschaftsgeld, er hat aber keinen unmittelbaren Zugriff auf das Familieneinkommen. Gibt der verdienende Ehegatte das Wirtschaftsgeld nicht freiwillig, so ist der andere Ehegatte gezwungen, den Klageweg zu beschreiten, was im „wahren Leben“ nur die wenigsten Ehegatten machen.

Mit dem Überschuss kann der verdienende Ehegatte tun, was ihm beliebt. Grenze ist nur der $\$ 1375$ BGB, wonach zum Endvermögen im Falle der Scheidung der Betrag hinzugerechnet wird, der der Vermögensverschwendung zuzurechnen ist. Dies bietet aber keinen hinreichenden Schutz, da die Vorschrift erst bei Beendigung des Güterstandes greift, keine Vermögensminderungen einbezieht, die länger als zehn Jahre her sind und der andere Ehegatte beweispflichtig für die Verschwendung ist.

Der haushaltsführende Ehegatte ermöglicht zwar erst durch seine Tätigkeit im Haushalt und der Kindererziehung die Berufstätigkeit des verdienenden, er hat aber keinerlei Recht auf Mitwirkung oder Teilhabe. Er ist völlig darauf angewiesen, dass der verdienende oder auch mehr verdienenden Ehegatte mit seinem Einkommen verantwortlich umgeht und dass er fähig ist, einen Zugewinn zu erwirtschaften. Ihm selbst sind in dieser Hinsicht die Hände gebunden. Was nutzt dann dem haushaltsführenden Ehegatten seine grundsätzliche wirtschaftliche Freiheit, wenn er kein Vermögen und kein Einkommen hat, um diese Freiheit auszuleben? Er hat während der Ehe nicht einmal einen konkreten Informationsanspruch hinsichtlich des Vermögens und des Einkommens. In einer Entscheidung hierzu hat sich der BGH auf den Standpunkt gestellt, in einer intakten Ehe würden sich die Ehegatten gegenseitig vertrauen, daher sei ein Unterrichtungsanspruch in der Ehe nicht notwendig. ${ }^{10}$ Demgegenüber hat der haushaltsführende Ehegatte in der Errungenschaftsgemeinschaft einen rechtlichen Anspruch auf die gemeinsamen Güter.

Bei der Beendigung der Ehe zeigt sich ein weiterer Mangel der Zugewinngemeinschaft. Bei der Errechnung des Endvermögens werden gemäß $\$ 1374$ BGB die vorehelichen Schulden nur bis zur Höhe des Anfangsvermögens abgezogen. Wenn einer der Ehegatten mehr Schulden als Anfangsvermögen hat, wird er so behandelt, als wäre sein Anfangsvermögen gleich Null. ${ }^{11}$ Hierdurch wird der Zugewinn des nichtverschuldeten
Ehegatten vermindert, ohne dass im Laufe der Ehezeit ein Ausgleich hierfür stattfinden würde. Während der Ehe wird dann der Teil des Endvermögens als nicht existent angesehen, den der verschuldete Ehegatte erwirtschaftet, wenn er damit voreheliche Schulden tilgt. Der weitere Nachteil des fehlenden Ausgleichs der Wertsteigerungen im Rahmen der Surrogation der Güter, $\mathbb{S} 1370$ BGB, wird durch die Reform ebenfalls beseitigt.

Das spanische Recht sieht einen Güterstand, in welchem ein Ehegatte keine Mitwirkungs- und Kontrollrechte hinsichtlich des in der Ehe erwirtschafteten Vermögens hat, als klar gleichheitswidrig an. Im deutschen Recht wird diese Frage kaum problematisiert. Es spricht aber einiges dafür, der spanischen Ansicht zu folgen und die Frage der Verfassungsmäßigkeit einiger Fragen innerhalb der Zugewinngemeinschaft zu stellen.

Insgesamt ist der Güterstand der Errungenschaftsgemeinschaft dem Güterstand der Zugewinngemeinschaft meines Erachtens vorzuziehen, der Grundgedanke des deutschen Güterrechts ist die Unabhängigkeit der beiden Ehegatten. Abgesehen davon, dass dies meiner Meinung nach grundsätzlich mit dem Grundgedanken der Ehe kollidiert, versagt er jedenfalls dann, wenn einer der Ehegatten aufgrund wirtschaftlicher Zwänge abhängig ist. Die Zugewinngemeinschaft ist zugeschnitten auf gleichberechtigte Ehegatten, die beide berufstätig sind und keine Kinder haben. Hier stellt sich dann die Frage, ob ein gesetzliches Güterrecht nicht seine Berechtigung verliert, wenn es lediglich auf die Ehen zugeschnitten ist, die die Minderzahl ausmachen. Beeinflusst durch den christlichen Gedanken ist die Vorstellung der Mehrheit der Bürger eine Ehe, die Kinder hervorbringt. Genau bei dieser Ehe aber zeigt die Zugewinngemeinschaft ihre größten Mängel. Im Übrigen ist es nicht die Ausnahme, sondern eher die Regel, dass die Scheidungswilligen beim Anwalt zu ihrer völligen Überraschung darauf hingewiesen werden müssen, dass sie entgegen ihrem bisherigem Glauben und Handeln eben nicht in einer Gütergemeinschaft leben und alles, was in der Ehe erworben wurde, beiden Ehegatten gemeinsam gehört, sondern in einer Gütertrennung. Zu diesem Zeitpunkt eröffnet sich dann auch häufig ein Informationsdefizit des Ausgleichsberechtigten, den das geltende Recht und auch die Reform nicht beseitigt. Wenn der ausgleichsberechtigte Ehegatte keine Informationen über das Vermögen des anderen Ehegatten bereits in der intakten Ehe gesammelt hat, gibt ihm der Auskunftsanspruch gegen den anderen Ehegatten nur ein stumpfes Schwert in die Hand. Ein echter eigener Auskunftsanspruch gegenüber Dritten wäre hier hilfreich.

\footnotetext{
10 BGH FamRZ 1976, $516 \mathrm{f}$.

11 Auch dies wird durch die geplante Reform nunmehr endlich behoben, siehe Fn 7 .
} 